\title{
ANÁLISIS DE PIEZAS ARQUEOLÓGICAS E IMAGINARIOS GOLEGTIVOS PARA EL DISEÑO DE PRODUGTOS ARTESANALES
}

\author{
ANALYSIS OF ARGHAEOLOGICAL PIEGES AND \\ COLLEGTIVE IMAGINARIES FOR THE DESIGN OF \\ HANDMADE PRODUGTS
}

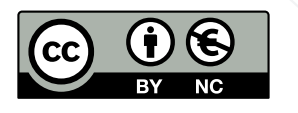

\author{
Michele Paulina Quispe Morales \\ Pontificia Universidad Católica del Ecuador sede Ambato \\ Ecuador
}

Diseñadora de profesión, graduada en la Facultad de Diseño de la Universidad del Azuay; desempeña las funciones docentes desde 2003 en instituciones como Universidad Técnica de Ambato, Universidad Cristiana Latinoamericana y Pontificia Universidad Católica del Ecuador - Ambato, dentro de diferentes áreas básicas y de especialidad. Cabe mencionar la participación en diversos cursos de capacitación dentro y fuera del país, tanto a nivel profesional como para el desempeño educativo a nivel superior, obteniendo así una Maestría en Tecnologías para la Gestión y Práctica Docente, y cursando actualmente otra en Diseño de Productos, mención en Innovación y Desarrollo de proyectos.

mquispe@pucesa.edu.ec

orcid.org/0000-0001-5905-276X 


\title{
Concepción del Carmen Bedón Vaca
}

Pontificia Universidad Católica del Ecuador sede Ambato Ecuador

Arquitecta Interiorista graduada en la Universidad Tecnológica Equinoccial en 2003. Estudios superiores en el área del diseño interior comercial en 2004. Magister en Tecnologías para la Gestión y práctica docente en 2015 y Diplomada en Diseño e Innovación social en 2017. Práctica Profesional desde 2002 en varios proyectos de tipología habitacional y comercial en las ciudades de Quito, Ambato, Puyo, Riobamba. Actividad docente desde el año 2004 en las universidades: Cristiana Latinoamericana, Técnica de Ambato, Cooperativa de Colombia y Pontificia Universidad Católica del Ecuador Sede Ambato desde 2010.

cbedon@pucesa.edu.ec

orcid.org/0000-0002-6963-4656

\author{
Abrahan Mora Pérez \\ Pontificia Universidad Católica del Ecuador sede Ambato \\ Ecuador
}

Ecuatoriano, Licenciado en Educación Básica de la Universidad Técnica Particular de Loja. Título de cuarto nivel Magister en Antropología y Cultura en la Universidad Politécnica Salesiana. Experiencia laboral docente en la Universidad Técnica de Ambato, Universidad Cristiana Latinoamericana y en la Pontificia Universidad Católica del Ecuador - Ambato, director, lector y tribunal de varios proyectos de titulación, tanto en pregrado como posgrado; coordinador de unidades académicas. Por otra parte, examinador del Programa de Bachillerato Internacional en Artes Visuales y Antropología. Además de la participación en varias investigaciones sobre temas culturales y adicciones con sus respectivas publicaciones. Así como la participación en congresos nacionales e internacionales.

amora@pucesa.edu.ec

orcid.org/0000-0003-2016-7222

Fecha de recepción: 20 de febrero, 2020. Aceptación: 08 de abril, 2020. 


\section{Resumen}

El presente documento se enfoca en los resultados obtenidos luego de un estudio formal y funcional de elementos, tanto del proceso de recolección catalográfico de varias piezas cerámicas encontradas en excavaciones dentro de la parroquia Sucre del cantón Patate de la provincia de Tungurahua en Ecuador -adjudicadas a las culturas Puruhá - Panzaleo, pertenecientes al período de integración regional y que reposan en su mayoría en la Tenencia Política de Sucre e Iglesia del Señor del Terremoto del cantón Patate- ; así como también, a los "imaginarios" del pueblo, los cuales revelan una identificación simbólica de la realidad y época de permanencia de la comunidad. Todo esto fundamentado en una investigación netamente etnográfica, de tipo cualitativo, que consintió la recopilación de discursos por medio de entrevistas y de la observancia de su actual estilo de vida para la interpretación y análisis del grupo en cuestión, considerando las relaciones de significado que se producen a nivel cultura o ideológico. De manera que el proceso de diseño aplicado, busca su rescate y revalorización, enmarcado en la descomposición de los mencionados compendios, pues la identificación de la esencia cultural, características y comportamientos propios de la zona, dan paso al desarrollo de nuevas propuestas alfareras con un toque identitario y modernista que permiten generar opciones de emprendimientos para la comunidad, con el propósito de lograr el desarrollo y bienestar de los habitantes de este sector.

\section{Palabras clave}

Puruhá - Panzaleo, Parroquia Sucre, artesanías, cerámica.

\section{Abstract}

This document focuses on the results obtained after a formal and functional study of elements; from the process of cataloging several ceramic pieces found in excavations inside the Sucre parish that belongs to Patate canton of Tungurahua Province in Ecuador - which is assigned to the Puruhá - Panzaleo cultures, belonging to the regional integration period and that mostly rests in Sucre's Political Tenure and the church "Iglesia del Señor del Terremoto" in Patate-; as well as the "imaginary" of people, which reveals a symbolic identification of the reality and time of permanence of the community. All this, based on purely qualitative ethnographic research which consisted of the collection of speeches through interviews and the observance of the current lifestyle for the posterior interpretation and analysis of the group at hand, considering also the relationships of meaning that are produced at cultural or ideological levels. Therefore, this applied design process, seeks its rescue and revaluation, framed in the decomposition of the aforementioned compendiums, since the identification of the cultural essence, characteristics, and behaviors of the area, give way to the development of new pottery proposals with an identity and modernist touch that allow generating entrepreneurship options for the community, with the purpose of achieving the development and well-being of the inhabitants of this sector.

\section{Keywords}

Puruhá - Panzaleo, Sucre Parish, handicrafts, pottery. 


\section{Introducción}

Las artesanías son un recurso importante dirigido a resaltar las expresiones de arte a través de variados materiales; en este caso nos centraremos en la cerámica. El objetivo de este artículo es visualizar las propuestas artesanales desarrolladas con integración de diseño, en las cuales se exterioriza la esencia de expresión cultural de la comunidad de Sucre.

Sin embargo, como menciona el Grupo Impulsor de Diseño Artesanal de México (2018), las menestralías se han convertido en un híbrido extraordinario, pues estos objetos ostentan una mezcla ceremonial, funcional y decorativa de gran valor cultural y económico para los pueblos. Lastimosamente, este sector se ha visto afectado por la ausencia del consumo de productos locales y/o tradicionales, ya que han sido sustituidos por las necesidades disímiles del consumista urbano, consecuentemente las nuevas generaciones artesanales tienen bajos ingresos económicos, lo que estimula al cambio de actividades y/o migración; por otro lado, está la demanda de productos importados con los cuales se disputa a nivel productivo, costo y variedad.

Por esta razón, el sector artesanal se ve inmerso en una constante evolución, que resulta de suma importancia para la integración cultural a través de los procesos de transformación de la artesanía, como parte del análisis previo al planteamiento de propuestas en las cuales se integra el diseño y la comercialización (Navarro, s/f). Adicionalmente es fundamental establecer su diferenciación con el arte, pues se dirige hacia la idea, hacia lo espiritual, mientras que la artesanía se asocia con lo sensible y placentero; dicho de otro modo, el arte se asocia al museo, mientras que la artesanía a la intimidad de los espacios (Grisales, 2015). En base a esta concepción, en el presente artículo, se consideran a los objetos artesanales desde una dimensión técnica, así como desde los niveles utilitario, estético y simbólico que otorga el diseño.

Es así que, como resultado final del proceso investigativo, se presenta esta última fase que se caracteriza por la inclusión de diseños estilizados en base al análisis formal y funcional de piezas cerámicas recuperadas en el sector de Sucre y a la interpretación discursiva de sus habitantes, sin dejar de lado su estilo de vida y costumbres; sirviendo esto de sustento para la elaboración de artesanías con diseños actuales, enmarcados en propuestas que integren la simbología del objeto y a la vez exploren nuevos imaginarios sociales.

\section{Las manifestaciones de los pueblos}

Analizar manifestaciones como la cerámica permite la comprensión de procesos culturales y modos de vida (Navarrete-Sánchez, 1990). A través de la identificación simbólica se perciben construcciones imaginarias de un grupo humano, de una etnia, en una realidad y época que determinan lo que constituyen una forma particular de ver e interpretar el mundo.

En el año 1989 se encontraron en el sector (parroquia Sucre en el cantón Patate perteneciente a la provincia de Tungurahua) piezas arqueológicas como vasijas, platos, adornos y huesos, que según datos investigativos del Patrimonio Cultural son pertenecientes a las culturas milenarias Panzaleo y Puruhá. Estos vestigios que al parecer corresponden a un mítico cementerio, reposan en su mayoría en la Tenencia Política de Sucre (La Hora, 2004). El problema identificado radica en el limitado análisis formal e iconográfico de dichos vestigios, lo cual ha impedido conocer los elementos simbólicos representativos de las culturas y su aporte para el desarrollo productivo, económico y cultural de la zona.

La finalidad del proyecto parte de generar un análisis e interpretación formal e iconográfica detallada de los ejemplares encontrados en el lugar, en búsqueda de una identificación analógica con las culturas antes mencionadas, para luego retomar dichos elementos característicos en la aplicación de elementos alfa- 
reros representativos de la zona, ya que esta técnica ancestral se está retomando como ente productivo de la comunidad para así contribuir con su desarrollo social, cultural y económico.

La búsqueda y rescate de identidades en el contexto ecuatoriano tiene actualmente una amplia trascendencia, lo que justifica plenamente esta investigación que tiene como objetivo estructurar nuevas alternativas artesanales integradas con el diseño, a través de un estudio étnico - cultural de los procesos simbólicos de la cerámica Puruhá de la parroquia Sucre que faciliten el desarrollo productivo de la comunidad.

\section{Proceso investigativo}

Resulta importante resaltar que el proceso investigativo se realizó en varias etapas; la primera, en la que se efectuó un registro catalográfico de las piezas de cerámica encontradas en la parroquia Sucre y que son adjudicadas al período de integración, el cual agrupa también un análisis formal detallado; un segundo momento está constituido por entrevistas a los longevos del pueblo, considerados por la comunidad como los entes sabios por su vivencia y experiencia, quienes aportaron con el discurso de sus relatos para luego indagar sobre sus imaginarios, dicha información fue reforzada con la observancia del contexto, recopilando elementos arquitectónicos y naturales para su exégesis; finalmente en tercera instancia, con la participación de los estudiantes de apoyo del proyecto, se generó la inclusión de diseños estilizados fundamentados en la abstracción e interpretación de los elementos mencionados, lo que servirá para la elaboración de artesanías con diseños actuales. Este artículo se ocupa específicamente de la tercera parte relacionada con la concepción de nuevos diseños.

Bajo los antecedentes ya mencionados, es importante resaltar que la relación entre el diseño y la artesanía, no tendría razón de ser, si no se ubica previamente el contexto, dirigido al lugar de emplazamiento y a las personas que lo habitan: su cultura, raíces y tradiciones. En este caso, el sitio de análisis es la localidad de Sucre, establecida como cabecera parroquial de Patate Urcu en la provincia de Tungurahua-Ecuador, cuya población es de 2369 habitantes y donde se localizó un gran patrimonio ligado a la memoria, herencia y por tanto a la pertenencia de la comunidad a través de vestigios cerámicos adjudicados a las etnias Panzaleo y Puruhá, que de acuerdo a Moreno (1988), habitaron tanto el altiplano como los flancos montañosos de las cordilleras.

Pensar únicamente en que la cerámica artesanal surgió por una necesidad, limita el desarrollo productivo y propositivo del diseño y su finalidad emocional y comunicativa; es por esto que se debe regresar en el tiempo y entender que los objetos parten de una sensibilidad arraigada en las personas y en su forma de vida. De manera general, se cuenta con evidencia que registra a las figuras encontradas en Sucre, dentro de la etapa tardía del período de integración de los Panzaleo y de los Puruhá (1140 -1500 D.C.). El Panzaleo está reconocido como una lengua preincaica hablada en los alrededores de la capital del Ecuador, antes de la conquista y mencionada por cronistas como Pedro Cieza de León, cuyo significado es "tribu o familia"; mientras que el nombre de Puruhá tiene algunos significados: puru = cerro y guay = casa grande. Sin embargo, su mayor significado se atribuye a la denominación de "lugar sagrado de permanencia", gracias al dios de los Puruháes (Pérez, 1970).

Centrándose en la cerámica de las etnias ya mencionadas, destacan las vasijas de uso doméstico como jarros, pondos y platos, las cuales sobresalen por sus formas de cabezas dramáticas y estilizadas que, de acuerdo a Pérez (1970), hacen alusión al poder y a la fertilidad. Los panzaleos destacan por la pintura negativa y positiva, el uso del puntuado en las compoteras y el modelado, características influenciadas por la ubicación y el comercio propio de las zonas de Pichincha, Tungurahua, Cotopaxi en la zona sierra, e incluso Quijos en la Amazonía ecuato- 
riana (Ontaneda, 2002). La cerámica puruhá es más gruesa y burda, sencilla en sus acabados, mas no en sus formas, caracterizada por elementos antropomorfos presentes en sus vasijas con cuatro narices y cuatro ojos.

Bajo este proceso de recolección, el diseño se vuelve consciente de la influencia del contexto en el desarrollo de nuevos productos, a través de la revalorización de lo ya existente sumado a una visión contemporánea de la realidad inmediata afín a la artesanía y la globalización. Es claramente palpable que, en un mercado basado en la competencia, difícilmente la artesanía pueda destacar entre los grandes capitalistas, por lo que la fusión con el diseño, se vuelve urgente y en el caso particular de esta investigación los pasos deben darse de forma continua a través del reconocimiento, la clasificación y el análisis de los vestigios encontrados.

La acción del diseño, por tanto, no es algo intempestivo, ya que el proceso se dirige a establecer un valor de marca, en este caso, centrado en los aspectos culturales de las etnias reconocidas y representadas a través de las vasijas, como un componente de la identidad de los colectivos que habitan el lugar, por lo que el objetivo es incorporar diseños de estas culturas ancestrales en una producción artesanal actual, con el fin de introducirlo en un mercado global.

\section{Metodología}

Es así que, como soporte para el desarroIlo del proyecto se maneja una investigación a nivel etnográfico y narrativo, lo que permitió ampliar la obtención y manejo de datos, pues el enfoque del trabajo está orientado a recolectar, analizar y clasificar información cualitativa, que, a pesar de ser un procedimiento netamente interpretativo y subjetivo, exige que los resultados obtenidos tengan validez y pertinencia, cumpliendo de esta manera la solidez científica necesaria.
Por otro lado, el diseño metodológico tiene un enfoque transversal de tipo descriptivo. Para la recolección de información se utilizaron técnicas como: observación participante, entrevistas a profundidad y revisión bibliográfica con un amplio trabajo de campo.

Para determinar las personas a las que se realizó las entrevistas se tomó en cuenta la exégesis de Víctor Turner, donde indica la triangulación de conocimientos entre personas de mayor estadía en la cultura, los nuevos miembros de la misma y el investigador (Turner, 1988). Además, la muestra en criterios de inclusión y exclusión. El análisis se ejecutó con etapas de codificación categorización, línea por línea y una posterior teorización (Poveda, Mora, Lara, \& Naranjo, 2016). Es así que, el análisis se complementa con el programa informático Zoon tropes (García, 2015). De esta información se obtiene que el imaginario de las personas entrevistadas que habitan en el lugar actualmente gira alrededor de: naturaleza, familia, dios y trabajo.

A nivel de diseño, es importante considerar la perspectiva que tiene el cliente de los productos, especialmente para que estos puedan competir en el mercado; es así que, nos centramos en el Diseño para la estética planteada por Macdonald (2001) en su artículo "Aesthetic intelligence: optimizing user-centred design".

Dicho autor enfatiza la vinculación de cualidades sensoriales a los valores culturales y propone un proceso de diseño que analiza los escenarios donde actúan los sentidos, zonas de tolerancia ambiental, escalas de medición, codificaciones, encuentros sensoriales, vista y empatía, toque y valor cultural; así como también los cambios en el valor cultural, señales emocionales y los sentidos, cultura y finalmente el diseño para los sentidos; con el fiel propósito de crear productos atractivos a los ojos del consumidor. 
Cabe recalcar, que el análisis catalográfico de las piezas de cerámica encontradas en el lugar y el discurso de los pobladores del lugar, realizados en la primera etapa, sirvieron para entender las sensibilidades de las personas y su forma de vida, lo que permitió proyectar a elementos de la naturaleza, familia, dios y trabajo, como parte del imaginario de los residentes de la parroquia.

\section{Propuesta de diseño}

Centrados en los aspectos culturales antes mencionados, se presentan fichas de análisis conceptual y formal, que permiten incorporar elementos simbólicos del contexto a una nueva producción artesanal de cerámica, con el fin de mejorar los productos para ser insertados en el mercado. Pues, este tipo de diseño, trata aspectos concernientes a la valoración de la belleza y de las emociones placenteras que este transmite en su uso.

Los resultados de esta tercera fase del proceso investigativo, se centra en el desarrollo de nuevas propuestas cerámicas que parten de los imaginarios de la población y del registro catalográfico de las piezas cerámicas: 
Tabla 1. Reconocimiento de la flor de zambo, descripción de sus características formales y propuesta

\section{ELEMENTO NATURAL DEL CONTEXTO: FLOR DE ZAMBO (CUCURBITA FICIFOLIA)}

\section{Fotografía}

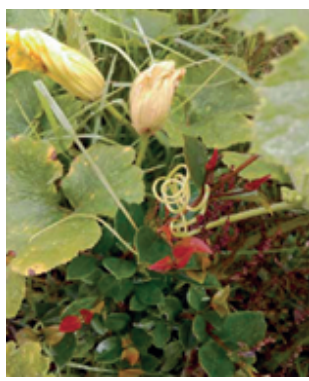

\section{Descomposición de elementos}

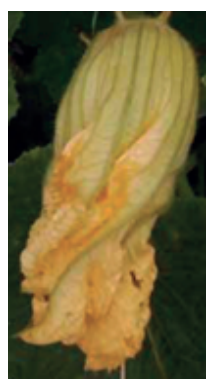

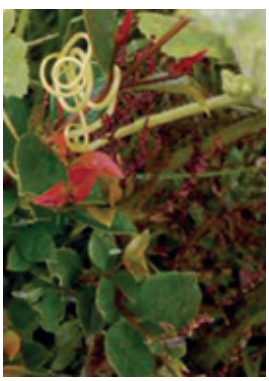

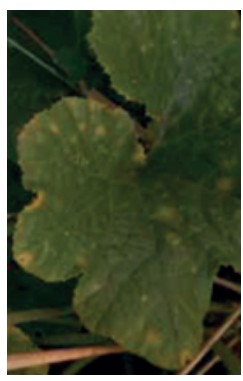

\section{Características}

Estas imágenes permiten visualizar los detalles que tienen la flor, hojas y demás componentes del zambo, sirviendo de inspiración para la creación de nuevas piezas cerámicas con líneas y cortes orgánico, mismos que fueron geometrizados para el mejor control de los procesos.

\section{Comportamiento}

Las propuestas de diseño creadas, parten de elementos orgánicos, pues la forma de inspiración posee un sinnúmero de líneas curvas muy pronunciadas, evidenciándose repetidamente la perfecta composición de la naturaleza.

\section{Colores}
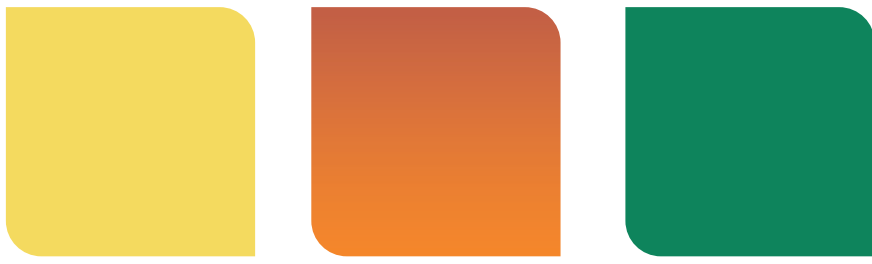


\section{Propuesta}

Contenedores basados en la extracción y estilización de formas de la flor del zambo.

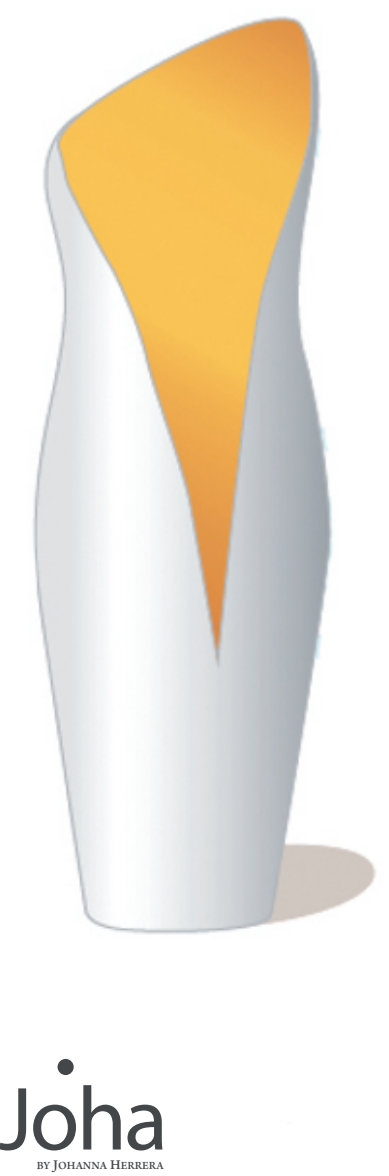

\section{SÜCRE}

EN Pontificia Universidad

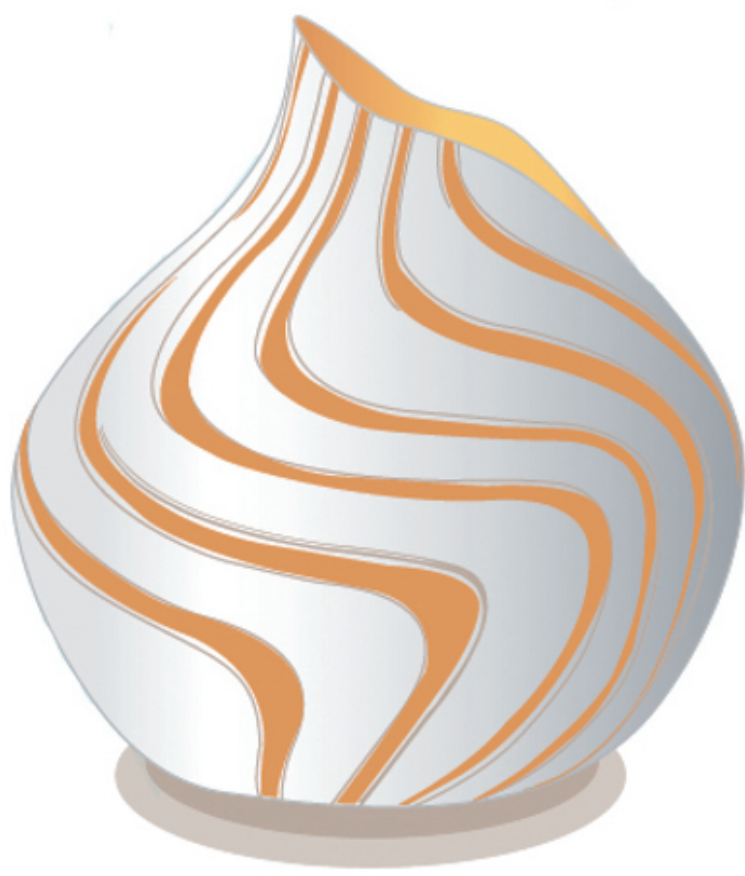

Fuente: Quispe, Bedón \& Mora, (2018).

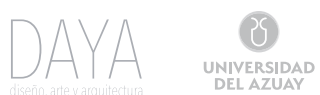


Tabla 2. Reconocimiento del maíz, descripción de sus características formales y propuesta

\section{ELEMENTO NATURAL DEL CONTEXTO: MAÍZ}

\section{Fotografía}

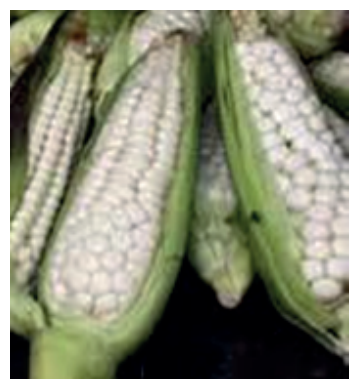

\section{Descomposición de elementos}
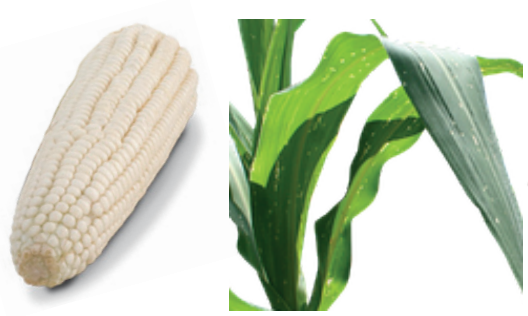

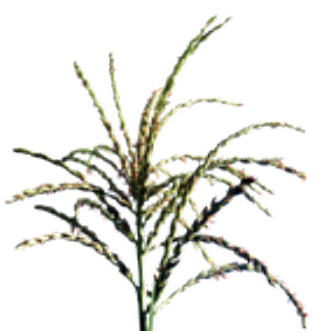

\section{Características}

Puede llegar a medir hasta casi tres metros de alto, es una planta robusta y sin ramificaciones. Su tallo es recto, cilíndrico y hueco.

Sus hojas son finas, largas, lanceoladas y un tanto onduladas.

Cada grano de maíz es un fruto totalmente independiente, inserto en un eje o raquis cilíndrico.

\section{Comportamiento}

Debido a la estructura del alto puede resistir fuertes vientos.

El valor nutricional que posee la mazorca es muy recomendable dentro de algunas dietas.

\section{Colores}
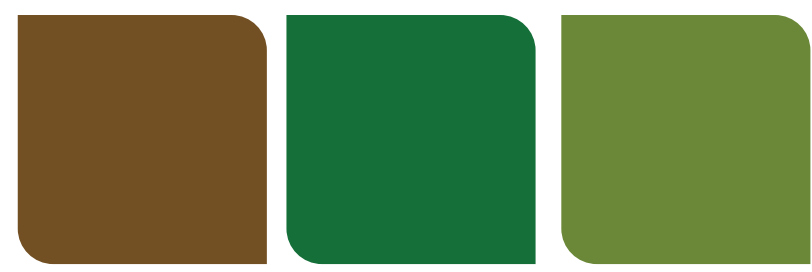


\section{Propuesta}

Contenedor y utensilio basados en la extracción y estilización de formas de la planta de maíz.

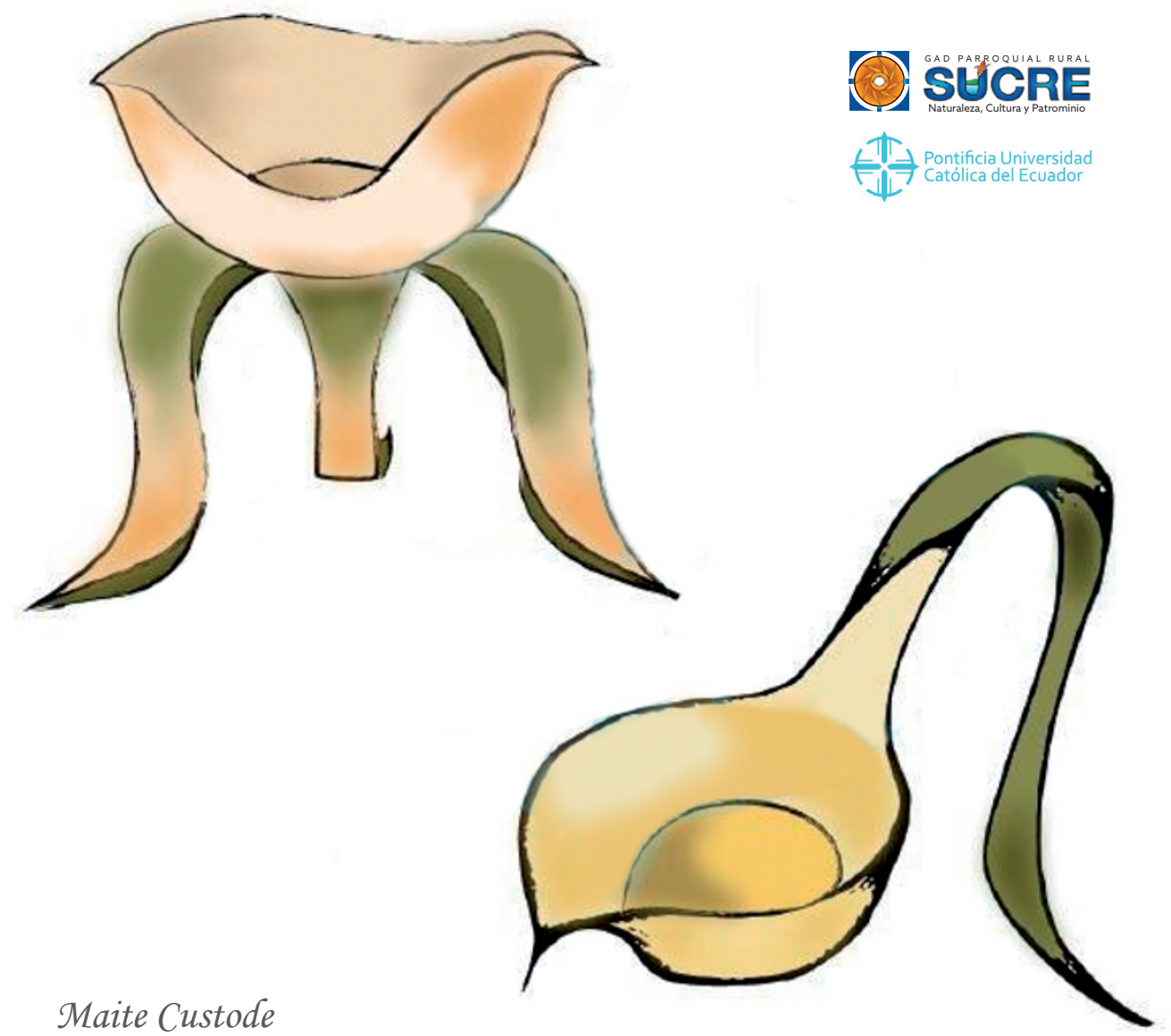

Fuente: Quispe, Bedón \& Mora, (2018). 
Tabla 3. Reconocimiento de la iglesia principal de la Parroquia Sucre, descripción de sus características formales y propuesta

\section{ELEMENTO CONSTRUIDO EN EL CONTEXTO: CÚPULA DE LA CAPILLA CENTRAL}

\section{Fotografía}

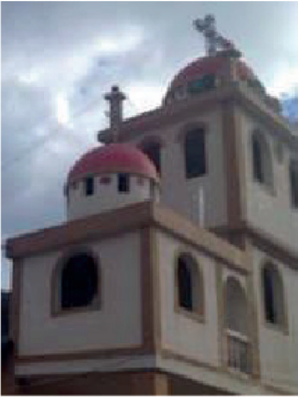

\section{Descomposición de elementos}

2
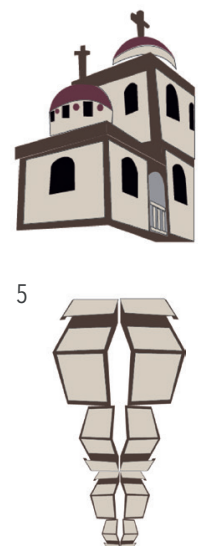

8

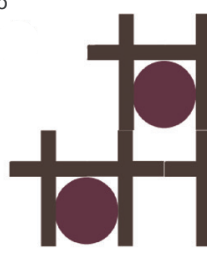

3

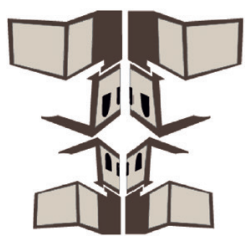

6

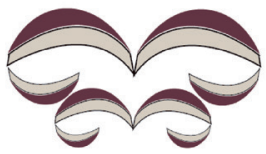

9

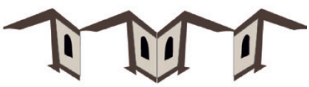

4

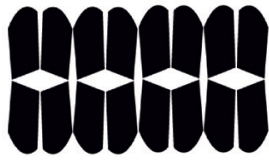

7

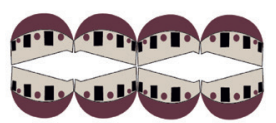

10

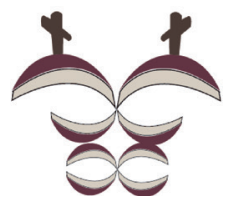

\section{Características}

Abarca diversas alternativas del credo del ministerio de la Palabra de Dios (evangelización, catequesis y homilía), para la formación espiritual de los habitantes de la parroquia; espacio referido para la celebración sacramental, especialmente de la eucaristía y oración.

\section{Comportamiento}

Infraestructura semimoderna, concebida en los años ochenta, como ente del enunciado católico; cuenta con una cúpula principal y un par de domos pequeños, su característica principal las formas en forma de arco de sus ventanas. 


\section{Colores}
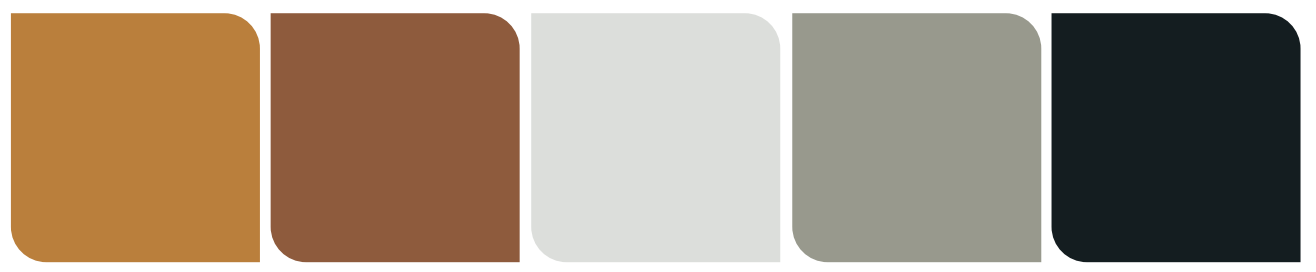

\section{Propuesta}

Contenedores basados en la extracción y estilización de formas de la iglesia parroquial.
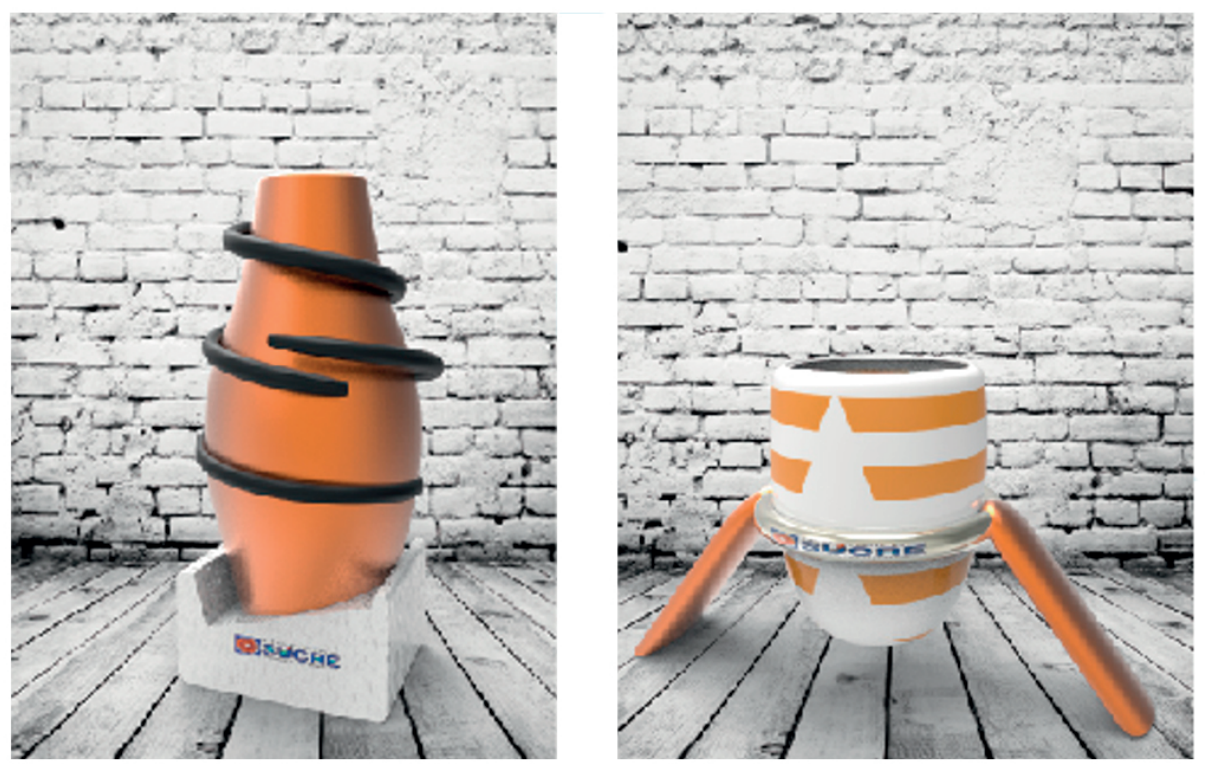

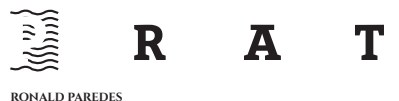

Fuente: Quispe, Bedón \& Mora, (2018).

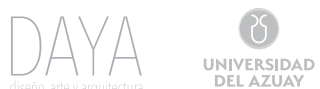


Tabla 4. Reconocimiento de los vestigios cerámicos, descripción de sus características formales y propuesta

\section{ELEMENTO GENERADO EN EL CONTEXTO: VASIJA}

Fotografía

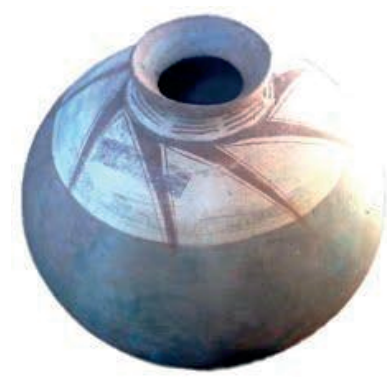

Descomposición de elementos

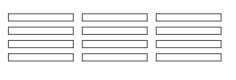

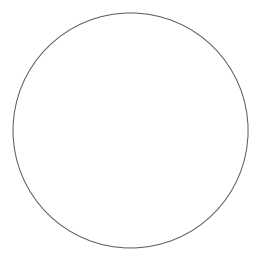

\section{Características}

Vasija de forma esférica que contiene una boca pequeña con respecto a su tamaño. Está dividida en tres partes: la primera ocupa más de la mitad del objeto de color gris sólido, la segunda por una parte más clara y con una iconografía triangular que se ubica en rotación alrededor del objeto y por último un conjunto de formas rectangulares en el cuello de la vasija.

\section{Comportamiento}

Se cree que se utilizó para el almacenaje de bebidas, alimentos o simplemente decoración.

\section{Colores}
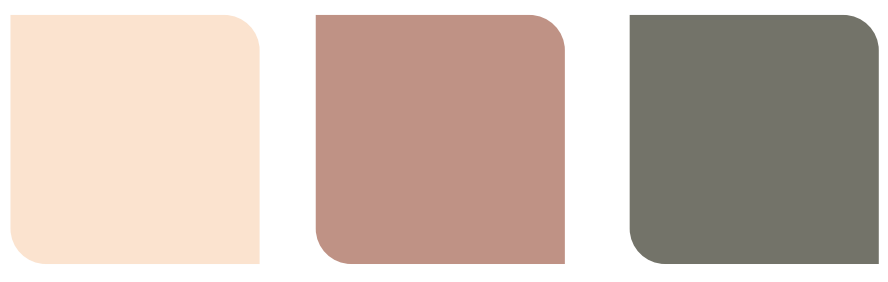


\section{Propuesta}

Compoteras basadas en la extracción y estilización de formas de los vestigios cerámicos.

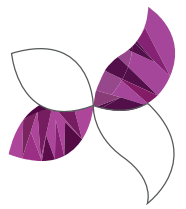

I Pontificia Universidad

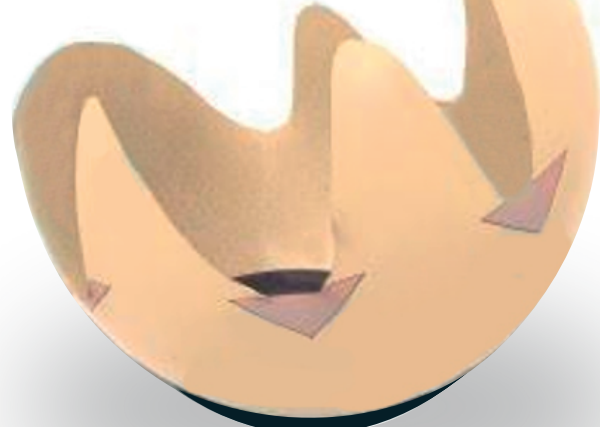

SUCRE

By July

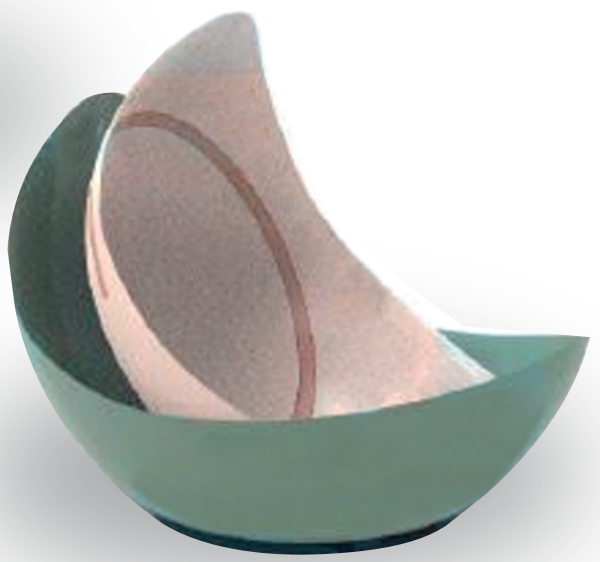

Fuente: Quispe, Bedón \& Mora, (2018). 
Tabla 5. Reconocimiento de los vestigios cerámicos, descripción de sus características formales y propuesta

\section{ELEMENTO GENERADO EN EL CONTEXTO: VASIJA}

Fotografía

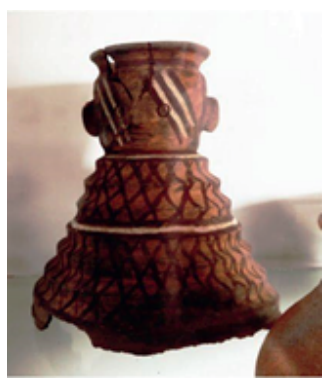

Descomposición de elementos
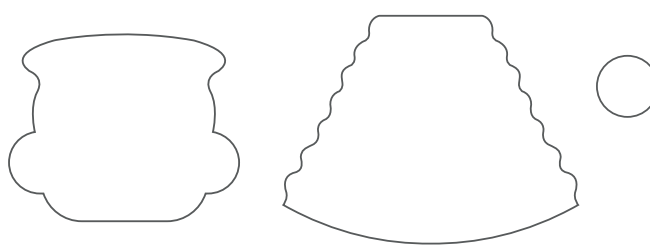
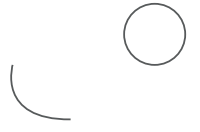

\section{Características}

Se acerca a la forma humana mediante la representación figurativa de un rostro, simplificándolo y geometrizándolo.

Es geométrico, predomina el uso de líneas en zigzag, tanto en la superficie como en el borde. Los colores se repiten a manera de franjas sobre el rostro y cuerpo de la cerámica.

\section{Comportamiento}

Se cree que se utilizó para el almacenaje de bebidas o alimentos, predomina la pintura, pues le otorga movimiento.

\section{Colores}
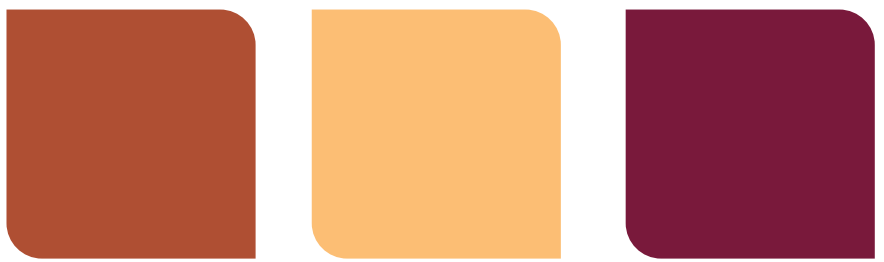


\section{Propuesta}

Compoteras basadas en la extracción y estilización de formas de los vestigios cerámicos.

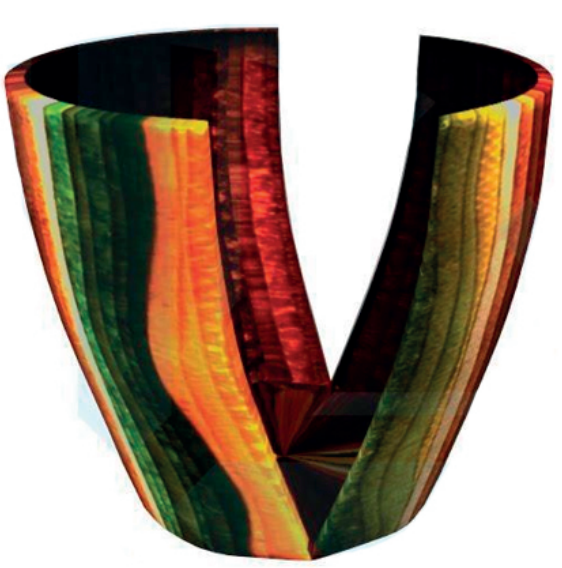

$\underset{H}{T} \Rightarrow$ Pontificia Universidac

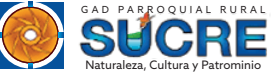
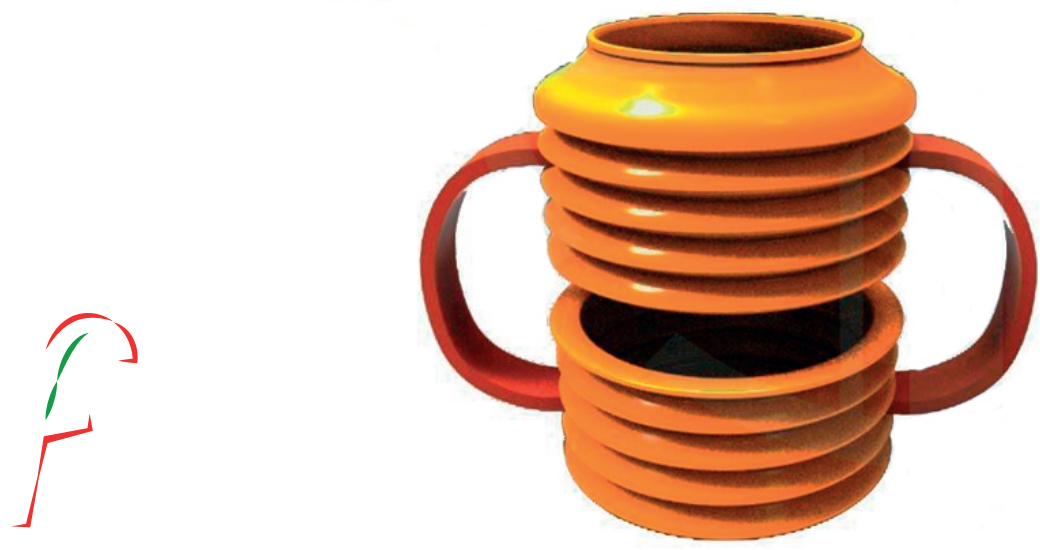

FRANCISCO COELLO

Fuente: Quispe, Bedón \& Mora, (2018). 
La innovación de productos permite mejorar su oferta, para dicho proceso deben ser considerados ciertos parámetros como la identidad morfológica, las características funcionales de los objetos, las necesidades y gustos, ente otros. Al analizar el aporte formal y funcional de las nuevas propuestas, se debe remarcar el manejo conceptual de los elementos propios de este pueblo, que fueron introducidos a nivel morfológico con el fin de generar productos artesanales identitarios para la zona.

Cabe recalcar que el trabajo cerámico realizado en Sucre, era una transcripción sin valor cultural. Razón por la cual, los niveles estéticos, simbólicos y funcionales fueron establecidos, en atención a los requerimientos del tipo de ferias en que los artesanos promocionan sus productos que son fomentadas por el gobierno provincial y visitadas en su mayoría por turistas; otro factor determinante para el establecimiento de propuestas fue la limitación de los procesos productivos, pues el régimen parroquial estaba dispuesto a introducir una artesanía que se había perdido en el tiempo, capacitándolos principalmente en la reproducción de piezas a través de moldes; es así que, se proponen artículos decorativos para el hogar, con miras a mejorar su comercialización e ingresos económicos.

Lastimosamente, el presente proyecto no pudo ver florecer sus frutos, pues el desarrollo del emprendimiento no fue factible debido al flujo migratorio de gran parte de su población, ya que en busca de maximizar sus réditos económicos se ha visto en la necesidad de abandonar su territorio de origen hacia destinos de concentración urbana, dejando este trabajo mancomunado sin continuidad.

\section{Conclusiones}

Del trabajo realizado se desprende que un proceso de diseño comunitario, que involucra la inclusión de los imaginarios, ya que la forma de vida de la población y su relación con el entorno, otorga la base necesaria para un desarrollo compositivo diferente, que mantiene las raíces ideológicas del pueblo; es así que, el diseño centrado en las personas, no solo se rige a una conversación estructurada, sino al registro de los elementos circundantes que permitieron establecer una revalorización de la cultura, que se encuentra perdida en el tiempo y difusa en su contexto.

Igualmente, se puede establecer que el diseño es una actividad compleja, que muchas veces debe partir de la descomposición de elementos, identificación de nuevas características y comportamientos, hasta llegar a la visualización final de formas, colores y texturas, pues estas deben ser manipuladas con sutileza y destreza para no perder su esencia en la necesidad de plantear nuevas propuestas; para lo que se vuelve imprescindible la participación de las comunidades, de forma que la integración e innovación no se dé únicamente en los nuevos elementos, sino también en las personas y en las nuevas alternativas de emprendimiento, con el propósito de expandir la intervención artesanal y productiva en la parroquia, para lograr así su desarrollo integral y por ende un progreso en su nivel de vida. 


\section{Referencias}

Foro Nacional de Artesanal Grupo Impulsor de Diseño Artesanal. (2018). Manual de Diseño y Desarrollo de Productos Artesanales. http://artesaniatextil.com/publicaciones/manual-de-diseno-y-desarrollo-de-productos-artesanales/

García, B. (2015). Análisis de las noticias sobre lectura digital: El País, El Mundo y abc (2012). Métodos de información (MEI), 57-85.

Grisales, A. L. (2015). Vida cotidiana, artesanía y arte. Thémata. Revista de filosofía, (51), 247-270.

La Hora (2004). Sucre guardó en sus entrañas a las culturas Panzalea y Puruhá. http://www.lahora. com.ec/index.php/noticias/show/1000239288/-1/Sucre_guardó_en_sus_entrañas_a_ las_culturas_Panzalea_y_Puruhá.html\#.Vh6ErOm9nIM

Macdonald, A. (2001). Aesthetic intelligence: Optimizing user-centred design. Journal of Engineering Design.

Mella, J. M. (2006). La innovación en la cerámica artesanal. Ministerio de Industria, Turismo y Comercio de España

Moreno, S. (1988). Formaciones políticas tribales y señoríos étnicos, Nuestra Historia del Ecuador. Quito: Corporación Editorial Nacional

Navarrete-Sánchez, R. (1990). Cerámica y etnicidad: una aproximación al estudio de las formas culturales como expresión de lo étnico. Boletín de antropología Americana, 47-80.

Navarro, S. La artesanía como industria cultural: Desafíos y oportunidades. Obtenido de Pontificia Universidad Javeriana, Departamento de Diseño, Facultad de Arquitectura y Diseño, Bogotá DC, Colombia, SA: https://www. fessociologia. com/files/congress/12/papers/3519. pdf.

Ontaneda, S. (2002). El Cacicazgo Panzaleo como parte del área circunquiteña. Banco Central del Ecuador. Pérez, A. (1970). Los Puruhuayes I. Quito: Casa de la Cultura Ecuatoriana.

Poveda, S., Mora, A., Lara, R., \& Naranjo, T. (2016). Predictores Clínicos de Demencia en Drogodependientes. Revista Neuropsicología, Neuropsiquiatría y Neurociencias, 16(3), 75-101.

Turner, V. (1988). El proceso ritual. Estructura y anti-estructura. Taurus.

Tablas

Tabla 1. Quispe, M., Bedón, C. \& Mora, A. (2018). Reconocimiento de la flor de zambo, descripción de sus características formales y propuesta.

Tabla 2. Quispe, M., Bedón, C. \& Mora, A. (2018). Reconocimiento del maíz, descripción de sus características formales y propuesta.

Tabla 3. Quispe, M., Bedón, C. \& Mora, A. (2018). Reconocimiento de la iglesia principal de la Parroquia Sucre, descripción de sus características formales y propuesta.

Tabla 4. Quispe, M., Bedón, C. \& Mora, A. (2018). Reconocimiento de los vestigios cerámicos, descripción de sus características formales y propuesta.

Tabla 5. Quispe, M., Bedón, C. \& Mora, A. (2018). Reconocimiento de los vestigios cerámicos, descripción de sus características formales y propuesta. 